\title{
Study tours: Nueva perspectiva del turismo académico en la universidad de Holguín
}

\author{
Study tours: New perspective of academic tourism at the University of \\ Holguín
}

M. Sc. Yaima Rodríguez Morales. ${ }^{1}$, M. Sc. Ernesto Batista Sánchez. ${ }^{2}$ \& Dr. C. David Almaguer La Rosa ${ }^{3}$

\begin{abstract}
The internationalization of higher education represents an alternative to disseminate the academic, scientific and cultural work of higher education institutions in the world and promote national and international exchange. Taking into account this trend, the University of Holguin, from its different areas, proposes and organizes various ways to internationalize its academic services. Among the modalities are the offers of international study programs, called Study Tours. The Study Tours are trips of an academic nature, where the teaching activities are intertwined with the sociocultural ones to create an attractive binomial for foreign students and professors interested in living the experience of knowing and learning from new cultures. With the aim of achieving greater international projection, the International Relations Directorate of the University of Holguín has developed the Study Tours programs: Tourism Management in Cuba, Cuban Language and Culture and Sociocultural Anthropology that will allow it to provide valuable educational and educational opportunities. Immersion in the Cuban environment and culture, students can also enrich their knowledge of places and people, as well as gain a deeper understanding of real-life situations and trends in the country and the proposed locations.
\end{abstract}

Key Words: Study Tours; Internationalization Of Higher Education; Academic Tourism.

\section{Resumen}

La internacionalización de la educación superior representa una alternativa para divulgar el quehacer académico, científico y cultural de las instituciones de educación superior en el mundo y promover el intercambio nacional e internacional. Teniendo en cuenta esta tendencia, la Universidad de Holguín, desde sus diferentes áreas, propone y organiza

\footnotetext{
${ }^{1}$ Universidad de Holguín, yayi@uho.edu.cu

${ }^{2}$ Universidad de Holguín, ernesto.batista@uho.edu.cu

${ }^{3}$ Universidad de Holguín, dri@uho.edu.cu
} 
variadas formas para internacionalizar sus servicios académicos. Dentro de las modalidades se encuentran las ofertas de programas internacionales de estudio, denominados Study Tours. Los Study Tours son viajes de carácter académico, donde las actividades lectivas se entrelazan con las socioculturales para crear un binomio atractivo para los estudiantes y profesores extranjeros interesados en vivir la experiencia de conocer y aprender de nuevas culturas. Con el objetivo de alcanzar una mayor proyección a nivel internacional, la Dirección de Relaciones Internacionales de la Universidad de Holguín ha elaborado los programas de Study Tours: Gestión Turística en Cuba, Lengua y cultura cubanas y Antropología sociocultural que le permitirán brindar valiosas oportunidades educativas y de inmersión en el ámbito y la cultura cubana, además los estudiantes pueden enriquecer su conocimiento de los lugares y las personas, así como obtener una comprensión más profunda de las situaciones y tendencias de la vida real en el país y las localidades propuestas.

Palabras Clave: Study Tours; Internacionalización De La Educación Superior; Turismo Académico.

\section{Introducción}

Para un óptimo desarrollo intelectual y para la formación de profesionales es cada vez más importante y necesario establecer conexiones entre las personas que se están formando y los profesores. Una forma de hacer esta premisa realidad es mediante el intercambio cultural y académico entre diferentes centros o instituciones formadoras, escuelas y universidades.

La internacionalización, en especial la movilidad internacional de estudiantes, ha transformado el panorama de la educación superior en la última década y ha traído diferentes beneficios a estudiantes e instituciones (Knight, 2012).

La internacionalización es un proceso que genera oportunidades a la universidad. De hecho, se trata de una poderosa herramienta para la mejora de la calidad, cuyos beneficios mejoran la competitividad de la universidad, primero, en el entorno nacional y, después, en el ámbito internacional. Actualmente, una de las tendencias con mayor boga es estudiar en el extranjero (study abroad), y dentro de ésta, la modalidad de Study Tours gana cada vez más fuerza y preferencia en el ámbito académico y estudiantil (Colectivo de autores, 2017).

Los Study Tours pueden ser categorizados de 3 maneras: estudios de un semestre completo en una institución extranjera, tours que involucran a los estudiantes en estancia de 1 semana a 1 mes en una universidad similar a la suya y la tercera es un tour internacional planificado de 2 a 3 semanas con conferencias de profesores de su Universidad para complementar los conocimientos adquiridos en la Centro donde se desarrollará el Study Tour (Hol et al., 2016).

De acuerdo con Scharoun, los 3 beneficios más relevantes de esta modalidad son: es una manera de interrelacionar a los estudiantes, que de otra manera quizás no pudieran viajar 
al extranjero; es una vía de incrementar la concientización e inteligencia intercultural y propicia el conocimiento desde sus experiencias en un escenario global (Scharoun, 2016).

Las bondades de esta modalidad se extienden también a los países que acogen a los estudiantes, entre ellos se pueden mencionar: incremento en la reputación del país y del sistema de educación superior debido a que los estudiantes pueden apreciar de primera mano la realidad que se vive y mostrar su experiencia a sus coetáneos una vez que regresen a sus países de origen. También el intercambio de estudiantes puede propiciar oportunidades de comercio, redes y relaciones entre las naciones implicadas (Ludlum, Ice, \& Sheetz-nguyen, 2013). Promueven, además, la tolerancia y el entendimiento de las culturas extranjeras. Las razones antes mencionadas producen "ciudadanos globales" capaces de tomar parte en una amplia gama de contextos culturales (Sison \& Brennan, 2012).

En la Universidad de Holguín (UHo) existen antecedentes de programas de intercambio. Entre ellos se puede mencionar el programa de intercambio sociocultural y comunitario que se realiza desde la Cátedra de Estudios Canadienses y la Universidad de Western Ontario, Canadá. El mismo se elabora específicamente para esta institución extranjera y se concilia de acuerdo a las necesidades y solicitudes de la misma basándose en relaciones ya establecidas a través de un convenio entre ambas instituciones. Pero como tal, no existen en la UHo antecedentes de un programa de Study Tours diseñado para su comercialización, de acuerdo a los estándares internacionales.

A través de diagnósticos que se han realizado como parte de disímiles investigaciones, se ha detectado como oportunidad el número y calidad de programas académicos que la UHo tiene para ofrecer, además de la disposición de sus áreas de estudios y profesores para brindar una educación superior de calidad. En este contexto y con el objetivo de alcanzar una mayor proyección a nivel internacional, la Dirección de Relaciones Internacionales (DRI) de la Universidad de Holguín en conjunto con diferentes Facultades, ha elaborado tres programas de Study Tours (Gestión Turística en Cuba, Lengua y cultura cubanas y Antropología sociocultural) con el fin de comercializarlos. Estos programas brindarán valiosas oportunidades educativas y de inmersión en la cultura cubana, además los estudiantes pueden enriquecer su conocimiento de los lugares y las personas, así como obtener una comprensión más profunda de las situaciones y tendencias contemporáneas en el país y las localidades propuestas.

\section{Desarrollo}

La elaboración de los programas se concibió en conjunto con las Facultades de la Universidad, donde cada área envió su propuesta académica (la cual debía ser interesante, diversa, y atractiva), y la Dirección de Relaciones Internacionales se encarga de organizar los detalles logísticos y la distribución de las actividades, tanto académicas como extracurriculares. Cada programa debe contar con un coordinador académico por parte de las Facultades, el cual debe fungir como intermediario entre ambas partes, además de asegurar y garantizar el óptimo desenvolvimiento todos los aspectos académicos. 
Para ello se realizaron diversas sesiones de trabajo entre los coordinadores de los programas de las Facultades y la DRI, así como con otras instituciones y organizaciones claves en el territorio involucradas en el desarrollo de estas ofertas académicas.

Los tres programas de Study Tours tienen una estructura general similar, pero difieren en cuanto a la temática académica, así como las excursiones y recorridos propuestos. Los mismos tienen dos modalidades: 1 semana (7 días) y/o 2 semanas (15 días). Para una mayor claridad, se expondrá el programa Gestión Turística en Cuba en su versión de 2 semanas.

\section{Objetivos del programa:}

De manera general, el programa está orientado a mostrar el funcionamiento del turismo en Cuba, y a brindar una perspectiva de los servicios que se ofertan en la modalidad de Sol y Playa.

- De forma más específica, los objetivos principales son:

- Conocer la historia del surgimiento del turismo en Cuba

- Comprender las principales diferencias del desarrollo turístico cubano en relación a los destinos competidores del Caribe

- Conocer el funcionamiento de las áreas fundamentales de un hotel

- Dirigir la operación de un restaurante o bar en una entidad turística

- Bailar los principales ritmos caribeños y diseñar un show de animación en hoteles

- Diseñar excursiones

- Elaborar productos turísticos tradicionales de Cuba

- Elaborar las estrategias de comercialización de los productos y servicios de destinos turísticos.

\section{Propuesta académica}

Las actividades académicas tienen un total de 45 horas, estructuradas en $25 \mathrm{hrs}$ de actividades lectivas y $20 \mathrm{hrs}$ de excursiones y recorridos especializados, las cuales guardarán relación con las temáticas impartidas en las mañanas. Las conferencias, al igual que los profesores que las ofrecerán, son propuestos por las áreas. Los programas de manera general tienen un enfoque integrador que, en el caso particular de Gestión Turística en Cuba, aunque el componente de mayor peso se centra en el turismo, no se obvian elementos indispensables como cultura y tradiciones cubanas que complementan y enriquecen la propuesta.

En la Tabla 1 se muestran las conferencias, excursiones y recorridos especializados asociadas en la propuesta. 
Tabla 1. Conferencias y excursiones

\begin{tabular}{|c|c|}
\hline Conferencias & Excursiones \\
\hline Introducción al turismo en Cuba & Visita a instalaciones extra hoteleras \\
\hline $\begin{array}{l}\text { Desarrollo del turismo en Cuba } \\
\text { (1492 - actualidad) }\end{array}$ & $\begin{array}{l}\text { Visita a las primeras instituciones turísticas de } \\
\text { Holguín (Mirador de Mayabe, Villa El Bosque y } \\
\text { Motel Pernik) }\end{array}$ \\
\hline Turismo Cultural & $\begin{array}{l}\text { Recorrido por los principales sitios de atractivo } \\
\text { turístico y cultural de la ciudad de Gibara }\end{array}$ \\
\hline Turismo patrimonial en Cuba & $\begin{array}{l}\text { Visita a museos y lugares de interés } \\
\text { arquitectónico de la ciudad }\end{array}$ \\
\hline Ritmos musicales cubanos & Apreciación de danzas tradicionales cubanas \\
\hline $\begin{array}{l}\text { Procesos básicos del turismo } \\
\text { (alojamiento, alimentación y bebida } \\
\text { y animación) }\end{array}$ & $\begin{array}{l}\text { Práctica de campo en instalaciones turísticas de } \\
\text { Sol y Playa }\end{array}$ \\
\hline $\begin{array}{l}\text { Gestión de agencias de viaje. } \\
\text { Diseño de excursiones }\end{array}$ & Visita a la Agencia de Viajes Havanatur \\
\hline Medio ambiente y turismo & $\begin{array}{l}\text { Visita al CITMA y exposición de los principales } \\
\text { proyectos relacionados con el cuidado del medio } \\
\text { ambiente en el área turística }\end{array}$ \\
\hline Turismo de naturaleza & Visita al Salto del Guayabo \\
\hline
\end{tabular}

\section{Tour - conferences}

Tour - conferences un término empleado por los autores para calificar un día completo de actividades académicas fuera de la Universidad, en otra municipalidad y/o provincia. En el caso del programa Gestión Turística en Cuba se propone la realización de 3 tour conferences:

- La primera se realizará en Gibará, donde se presentará la Conferencia: Turismo cultural que se desarrollará en el Salón de Reuniones del cine Jibá. Y en la tarde se hará una excursión por los principales sitios turísticos de la ciudad y una visita al parque eólico donde se podrá apreciar una panorámica del trabajo del municipio en materia de energías renovables. Se escogió Gibara para esta conferencia pues la ciudad es un referente en cuanto a cultura y tradiciones. Es sede de numerosos eventos a lo largo del año, uno de los más famosos es el Festival de Cine en Gibara, donde realizadores de diversas partes del mundo se reúnen para mostrar su quehacer fílmico. Además, su arquitectura y belleza es de un atractivo irresistible para los visitantes, tanto nacionales como extranjeros.

- Tomando en cuenta los principales objetivos del Study Tour, el segundo tour conference se realizará en el Polo Turístico, en horas de la mañana se impartirá Procesos básicos del turismo, y en la tarde se complementará la lección con una práctica de campo sobre lo aprendido en la conferencia, donde se podrá apreciar e interactuar con los procesos de alojamiento, alimentación y bebidas y animación en instalaciones turísticas de Sol y Playa. 
- Para finalizar el ciclo de tour -conferences, Turismo de naturaleza, que se desarrollará en Pinares de Mayarí. En Pinares se encuentra la instalación que tiene el mismo nombre de la zona, donde su mayor atractivo es la excursión al Salto del Guayabo, lugar rico en biodiversidad y donde se podrá apreciar cómo se maneja esta rama del turismo.

Los beneficios de las tour - conferences radican en que los estudiantes pueden adquirir conocimientos valiosos fuera del salón de clases, lo cual es atractivo y provechoso. Además, pueden apreciar y comprobar lo aprendido en el mismo lugar donde se imparte la conferencia e interactuar directamente con el ambiente que los rodea.

\section{Excursiones}

Las excursiones se planificaron con el objetivo de que guarden relación directa con la temática que se trate durante la mañana. Por ejemplo, cuando se imparta la conferencia sobre Turismo patrimonial y cultural en Cuba, la excursión relacionada será la visita guiada a museos y sitios de valor cultural y patrimonial de la ciudad. En el caso de la conferencia Medio ambiente y turismo, en la tarde se realizará un recorrido por el CITMA, donde se podrá apreciar una exposición de los principales proyectos en ejecución relacionados con la protección al medio ambiente en la esfera turística, por solo exponer estos ejemplos.

\section{Fin de semana}

El fin de semana es libre (on their own). Se les gestionarán ofertas atractivas que contribuyan a reforzar el programa académico propuesto. Estos Weekend Tours incluyen ofertas de transportación y hospedaje en el lugar de destino. Teniendo en cuenta la cercanía geográfica y la riqueza cultural, patrimonial e histórica se sugieren: Santiago de Cuba y el Polo turístico de Holguín, con sus hermosas playas e instalaciones. Otra opción puede ser disfrutar de un fin de semana en la ciudad, donde se les facilitará un programa cultural para que puedan conocer las opciones que brindan los principales centros culturales y recreativos. Es importante destacar que en todos los momentos estos programas deben propiciar el intercambio con estudiantes de las carreras de la universidad que tributen al Study Tour.

\section{Transportación}

Se propone que la transportación se realice, en su mayor parte, contando con los servicios de agencias de turismo, como Transtur, con el fin de brindar un mayor confort. En el caso de los Weekend Tours fuera del municipio, se brinda la alternativa del traslado en autos antiguos, lo cual se debe al alto atractivo turístico que estos carros ofrecen a los clientes foráneos. 


\section{Estancia}

Se presenta la opción de realizar la estadía en casas de renta particulares que permiten al estudiante adquirir la experiencia de convivir con una familia cubana y tener un contacto más cercano con sus habitantes.

\section{Flexibilidad}

De acuerdo con los intereses de la parte extranjera, se puede gestionar el cambio, adición o sustracción de alguna temática. Se puede incluir también conferencias de algún profesor extranjero que asista como acompañante al Study Tour.

\section{¿Por qué elegir a Holguín para una estancia de Study Tour?}

Primeramente, por la calidad de su institución rectora. La Universidad de Holguín fue fundada hace 50 años, ubicada en la capital de la provincia. Oficialmente acreditada y reconocida por el Ministerio de Educación Superior, está compuesta por 4 campus y 13 centros universitarios municipales, donde se ofrecen diversos cursos y programas. $\mathrm{Su}$ misión fundamental es asegurar la competencia de los profesionales comprometidos con el desarrollo y participación en los procesos de una sociedad en crecimiento.

La Universidad de Holguín cuenta con 8 facultades donde radican 49 carreras en cursos diurnos, por encuentro y a distancia, 14 de ellas acreditadas, 5 con categoría de excelencia por la Junta de Acreditación Nacional. Existen también 13 programas de Maestrías, Programas de Especialidades de Postgrado, 10 Programas de Doctorado y 7 centros de estudios que tienen como objetivo el elevar la formación científica, académica y profesional de los clientes en diversas ramas de las ciencias.

Claustro: los profesores que participarán en el programa poseen vasta experiencia en temas turísticos. Entre el claustro se pueden encontrar directivos de entidades turísticas, lo cual propicia el enriquecimiento de los conocimientos teóricos con la experiencia de la puesta en práctica.

Holguín: de manera general la provincia está especialmente bendecida por la naturaleza que la dotó de una riqueza paisajística sorprendente y espectaculares playas de fama internacional. Pero tan impresionante como sus valores naturales son sus tradiciones e historia. Baste decir que su puerto de Bariay es el sitio donde desembarcó Cristóbal Colón por primera vez en Cuba y lugar de nacimiento de Iberoamérica.

Ciudad: su aspecto urbano está dominado por varios parques, es por ello que es reconocida como "la Ciudad de los Parques". Por la exquisita y rigurosa conservación de la estructura arquitectónica colonial que poseen, los parques de Holguín, son, además, como un hermoso pulmón de la identidad local urbana. Es una ciudad muy cultural, anualmente tienen lugar en Holguín diversos espacios artísticos y culturales donde se pueden destacar: Romerías de Mayo, Semana de la Cultura Iberoamericana, Festival Nacional de Música Electrónica Stereo - G, Concurso de Danza del Atlántico Norte 
Codanza y Grand Prix Vladimir Malakhov, Encuentro Nacional de Agrupaciones Soneras, por solo mencionar algunos.

Polo turístico: en su costa norte Holguín posee atractivos inigualables. 110 kilómetros decorados por siete impresionantes bahías y 23 kilómetros de espectaculares playas, varias de excepcionales calidad y hermosura. Precisamente en esta franja costera se localizan los principales resorts, los parques naturales más importantes de Holguín, los cuales cuentan con una amplia gama de servicios dirigidos a atraer consumidores de todas partes del mundo. El producto turístico clásico que distingue al destino Holguín es el de sol y playa, que además de explotar una de sus ventajas naturales, constituye aún el principal atractivo para los turistas que lo visitan. Razón por la cual se erige como escenario perfecto para el aprendizaje del manejo de los principales procesos turísticos propuestos en el programa.

\section{Precio}

La Universidad de Holguín realizará el cobro solo por las actividades académicas. El precio por estudiante dependerá entonces de la cantidad de horas de actividades lectivas y de excursiones y recorridos especializados que estén planificadas en el programa en el que decida participar. El precio estipulado por hora de conferencia es de $25 \mathrm{CUC}$, y 20 CUC en el caso de las demás actividades (excursiones y recorridos especializados).

En el caso particular de Gestión Turística en Cuba, al poseer el programa 25 horas programadas de conferencias y 20 de las demás actividades, el costo sería un aproximado de 1025 CUC por participante. Se admiten grupos a partir de 5 estudiantes y al menos un profesor o responsable de la institución de los mismos.

Los precios pueden ser negociados de acuerdo a los intereses de las dos instituciones.

\section{Programas Lengua y cultura cubanas y Antropología cultural}

Se considera, por parte de los autores, oportuno ofrecer información acerca de los otros programas que brinda la Universidad de Holguín y mostrar una breve descripción de los mismos, así como sus principales beneficios.

Lengua y cultura cubanas: El curso está dirigido a mostrar una panorámica de la lengua española en Cuba, sus orígenes y vertientes, además de la fraseología típica de nuestra nacionalidad. Se podrá conocer elementos distintivos de la cultura y tradiciones cubanas, además de hacer un recorrido por las principales manifestaciones culturales presentes en la ciudad de Holguín. Con este programa adicionalmente se logrará:

- Practicar el idioma español

- Obtener un enriquecimiento cultural e idiomático

- Lograr inmersión en la cultura cubana

- Visitar lugares vinculados a la historia y la cultura cubanas

- Apreciar las principales manifestaciones artísticas cubanas 
Antropología sociocultural: Este programa brinda una visión de la génesis de los principales procesos culturales y sociales del país, enfocándose en la ciudad de Holguín. Presenta a los cursistas las particularidades del sistema educativo cubano y de los procesos de desarrollo local y estudios de género. Al cursar este programa se podrá conseguir:

- Conocer las características del sistema económico cubano

- Lograr inmersión en las tradiciones cubanas

- Intercambiar con la comunidad

- Apreciar las principales manifestaciones culturales cubanas

- Visitar lugares de atractivo histórico y cultural

\section{Conclusiones}

- La internacionalización de la Educación Superior permite el intercambio académico nacional e internacional. En este sentido, los Study Tours brindan a los estudiantes la oportunidad de conocer y aprender de nuevas culturas, además de ser una vía de incrementar la concientización e inteligencia intercultural y propicia el conocimiento desde sus experiencias en un escenario global. El análisis desarrollado demuestra que en la Universidad de Holguín no existían programas de Study Tours para su divulgación y comercialización, por lo que se desarrollaron tres programas de Study Tours en las áreas de mayor fortaleza académica (Antropología sociocultural, Lengua y Cultura cubanas y Gestión Turística).

\section{Referencias bibliográficas}

Colectivo de autores. (2017). Estrategia de internacionalización de la Universidad de Málaga.

Hol, A., Simiana, D., Lieu, G., Ong, I., Feder, J., Dawre, N., \& Almazi, W. (2016). International Study Tours: a key to 21 st century academic and industry exchange. International Conferences ITS, ICEduTech and STE (pp. 213-220).

Knight, J. (2012). Student Mobility and Internationalization: trends and tribulations. Comparative and International Education, 7(1), 20-33. doi:10.2304/rcie.2012.7.1.20

Ludlum, M., Ice, R., \& Sheetz-nguyen, J. (2013). Justifying Study Abroad in Financially Difficult Times. Administrative issues journal: education, practice and research, 3(2). doi:10.5929/2013.3.2.6

Scharoun, L. (2016). Short-term study tours as a driver for increasing domestic student mobility in order to generate global work-ready students and cultural exchange in Asia Pacific. Perspectives: Policy and Practice in Higher Education, 20(2), 83-89. doi:10.1080/13603108.2015.1075917 
Sison, M. D., \& Brennan, L. (2012). Students as global citizens : strategies for mobilizing studies abroad, 1241. doi:10.1080/08841241.2012.736884 


\section{PARA CITAR EL ARTÍCULO INDEXADO.}

Rodríguez Morales, Y., Batista Sánchez, E., \& Almaguer La Rosa, D. (2021). Study tours: Nueva perspectiva del turismo académico en la universidad de Holguín. Explorador Digital, 5(1), 218-228. https://doi.org/10.33262/exploradordigital.v5i1.1499

\section{¿Ciencia}

El artículo que se publica es de exclusiva responsabilidad de los autores y no necesariamente reflejan el pensamiento de la Revista Explorador Digital.

El artículo queda en propiedad de la revista y, por tanto, su publicación parcial y/o total en otro medio tiene que ser autorizado por el director de la Revista Explorador Digital.
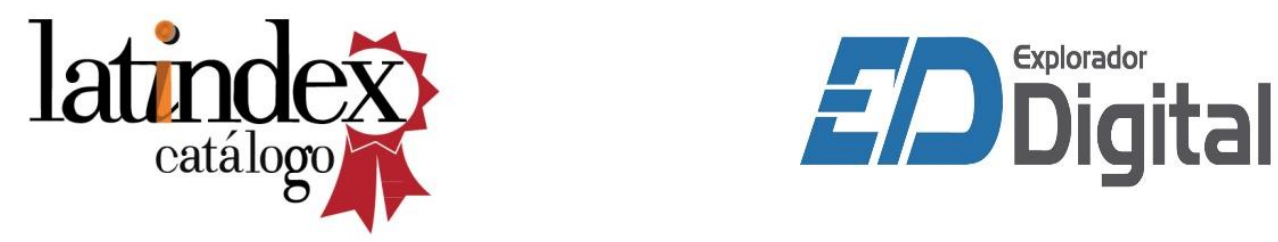